\title{
Corrigendum: A Pharmacogenomic-based Antidepressant Treatment for Patients with Major Depressive Disorder: Results from an 8-week, Randomized, Single-blinded Clinical Trial
}

\author{
Changsu Han ${ }^{1}$, Sheng-Min Wang ${ }^{2,3}$, Won-Myong Bahk ${ }^{2}$, Soo-Jung Lee ${ }^{2}$, Ashwin A. Patkar ${ }^{4}$, Prakash S. Masand ${ }^{5}$, \\ Laura Mandelli ${ }^{6}$, Chi-Un Pae ${ }^{2,4,7}$, Alessandro Serretti ${ }^{6}$ \\ ${ }^{1}$ Department of Psychiatry, Korea University College of Medicine, Seoul, ${ }^{2}$ Department of Psychiatry and ${ }^{7}$ Cell Death Disease Research Center, \\ College of Medicine, The Catholic University of Korea, Seoul, ${ }^{3}$ International Health Care Center, Seoul St. Mary's Hospital, College of Medicine, \\ The Catholic University of Korea, Seoul, Korea, ${ }^{4}$ Department of Psychiatry and Behavioral Sciences, Duke University Medical Center, Durham, \\ NC, ${ }^{5}$ Global Medical Education, New York, NY, USA, ${ }^{6}$ Department of Biomedical and Neuromotor Sciences, Psychiatric Section, University \\ of Bologna, Bologna, Italy
}

Clin Psychopharmacol Neurosci 2018;16(4):469-480

The authors found inadvertent errors including typos in the above published paper. In the baseline comparison, typos are corrected and all items of categorical parameters are presented to ensure the proportion. $P$ values are partly changed to correct oversight on statistics from Chi-square, Fisher's exact test, and nonparametric test. As for comparison of continuous clinical outcomes between the two groups, there was an error in proper inclusion of covariates proposed in the original version. As for comparison of proportional outcomes between the two groups, there were subjects not included in the analysis (response comparison, 5 for PGATx and 9 for TAU; remission comparison, 7 for PGATx and 9 for TAU; CGI response comparison, 6 for PGATx and 2 for TAU; FIBSER comparison, 13 for PGATx and 18 for TAU). Therefore, the results of Tables $1 / 2$ and Figures $2 / 3$, and corresponding paragraphs within the text are all corrected. However, all corrected results are in line with the original outcomes without any directional changes and thus these corrections do not change the scientific conclusions of the paper in any way. The authors would like to deeply apologize for inadvertent errors that appeared in the paper.

A revised version of this paper containing the corrections noted above has been published as supplementary data (see uploaded file).

Supplementary Data: 1. Correction. 2. Corrected version of the originally published paper.

\footnotetext{
Address for correspondence: Chi-Un Pae

Department of Psychiatry, The Catholic University of Korea, Bucheon St. Mary's Hospital, 327 Sosa-ro, Wonmi-gu, Bucheon 14647, Korea E-mail: pae@catholic.ac.kr

ORCID: https://orcid.org/0000-0003-1632-4248
}

(c) This is an Open-Access article distributed under the terms of the Creative Commons Attribution Non-Commercial License (http://creativecommons.org/licenses/by-nc/4.0) which permits unrestricted non-commercial use, distribution, and reproduction in any medium, provided the original work is properly cited. 